\title{
L'écologie industrielle : quand l'écosystème industriel devient un vecteur du développement durable
}

\section{Arnaud Diemer et Sylvère Labrune}

\section{(2) OpenEdition}

1 Journals

\section{Édition électronique}

URL : http://journals.openedition.org/developpementdurable/4121

DOI : 10.4000/developpementdurable.4121

ISSN : 1772-9971

Éditeur

Association DD\&T

\section{Référence électronique}

Arnaud Diemer et Sylvère Labrune, "L'écologie industrielle : quand l'écosystème industriel devient un vecteur du développement durable », Développement durable et territoires [En ligne], Varia (2004-2010), mis en ligne le 30 août 2007, consulté le 10 décembre 2020. URL : http://journals.openedition.org/ developpementdurable/4121; DOI : https://doi.org/10.4000/developpementdurable.4121

Ce document a été généré automatiquement le 10 décembre 2020.

Développement Durable et Territoires est mis à disposition selon les termes de la licence Creative Commons Attribution - Pas d'Utilisation Commerciale 4.0 International. 


\title{
L'écologie industrielle : quand l'écosystème industriel devient un vecteur du développement durable
}

\author{
Arnaud Diemer et Sylvère Labrune
}

1 La prise de conscience de la portée des problèmes écologiques de la planète a pris une dimension mondiale depuis la conférence des Nations Unies sur l'environnement humain, organisée en 1972 à Stockholm. Les années qui ont suivi ont vu la naissance d'un mouvement écologique international, l'émergence de milliers d'organisations écologiques locales et la prolifération dans bien des pays à travers le monde de lois et de réglementations concernant l'environnement. La fin du XX ${ }^{\text {ème }}$ siècle a toutefois constitué le théâtre d'affrontements entre deux disciplines en complète opposition, l'économie et l'écologie (Vivien 1994).

2 Les économistes analysent l'évolution de la croissance (principalement le PIB) en s'appuyant sur des estimations de la consommation des ménages, de l'investissement des entreprises, du déficit public et des termes de l'échange (exportations, importations). Leur point de vue prédomine dans le monde de l'industrie et des finances, ainsi qu'au sein des gouvernements et des agences internationales de développement (Banque Mondiale, FMI, CNUCED...). L'écologie ne représenterait qu'une sous discipline mineure de l'économie (certains l'associentà l'économie des ressources naturelles et/ou l'économie de l'environnement) qui doit être intégrée dans les modèles (Diemer 2005). Les écologistes étudient quant à eux les rapports complexes et toujours changeants qui lient les organismes vivants à leur environnement. Cette vision, rappelle Nicholas GeorgescùRoegen (1977a, p. 13) procède de «la biologie et de la thermodynamique »mais est également influencée par d'autres disciplines telles que l'agronomie, l'hydrologie, la démographie... Pour l'écologiste, la croissance est limitée par les paramètres de la biosphère. L'économie ne serait qu'un secteur étroit de l'écosystème global. Les activités économiques croissantes de l'humanité ne pouvant être séparées des ressources et des systèmes 
naturels dont elles dépendent en dernier ressort, toute activité qui sape l'écosystème global ne pourrait se poursuivre indéfiniment.

3 Longtemps séparées, les questions d'économie et d'écologie sont devenues inextricablement liées pour définir ce que l'on appelle aujourd'hui le développement durable (Brunel 2004; Bourg, Rayssac 2006). Cette expression est entrée dans le vocabulaire courant à la suite du rapport Brundtland (1987) - le développement durable correspond au " développement qui répond aux besoins du présent sans compromettre la capacité des générations futures de répondre aux leurs » - mais, pour beaucoup ${ }^{1}$, le sens et l'apport du développement durable restent encore obscurs (Bourg 2002; Smouts et al. 2005). Deux courants de pensée sont généralement évoqués lorsqu'il s'agit de revenir sur les origines de ce concept (Dannequin, Diemer, Vivien 1999a). Le premier se range sous la bannière de l'écologie industrielle (Frosch, Gallopoulos 1989, Erkman 1998). Le second, plus difficile à cerner, puisqu'il n'apparaît pas en tant que tel, regroupe un certain nombre d'auteurs, comme Ivan Illich $(1973,1975)$, André Gorz $(1978,1988)$ ou Nicholas Georgescu-Roegen (1978, 1993), que l'on range dans les rangs de l'écologie politique (Bourg 1996; Dannequin, Diemer, Vivien 1998).

4 L'objet de cet article est de clarifier le concept de développement durable en l'examinant plus particulièrement sous l'angle de l'écologie industrielle². Les tenants de l'écologie industrielle (Ayres 1993, Frosch 1995, Graedel 1996, Erkman 1998) ont précisé que cette dernière se proposait de donner un contenu opérationnel à la notion de développement durable. Robert Frosch (1995, p. 148) associe l'écologie industrielle à "l'ensemble des pratiques destinées à réduire la pollution industrielle » et Suren Erkman (1998) a matérialisé cette idée par un sous-titre évocateur « mettre en pratique le développement durable dans une société hyper-industrielle ». L'écologie industrielle peut être présentée comme une nouvelle pratique du management environnemental. Elle répond aux besoins des entreprises, qui sous la pression des lois, des règlements, des normes et de la concurrence, cherchent à intégrer l'environnement à leurs stratégies. L'écologie industrielle (Frosch, Gallopoulos 1989, Erkman 1998), l'ingénierie écologique ou l'écotechnologie (Mitsch, Jorgensen 1989) recommandent ainsi aux industriels de procéder à un ensemble d'opérations de rationalisation de la production (optimisation des consommations énergétiques et matérielles, minimisation des déchets à la source,réutilisation des rejets pour servir de matières premières à d'autres processus de production). L'écosystème industriel deviendrait dès lors un véritable vecteur du développement durable.

5 Afin de saisir la réelle portée de ce programme, nous procéderons en deux étapes. Dans un premier temps, nous évoquerons la démarche et les grands principes de l'écologie industrielle. De nature interdisciplinaire,l'écologie industrielle donne une vision globale et systémique des interactions entre la société industrielle et la biosphère. Elle s'appuie principalement sur le métabolisme industriel (analyse des flux de matières, bilans matière-énergie, matrice input-output, évaluation du cycle de vie...) et les lois énergétiques du monde physique. Dans un second temps, nous présenterons la symbiose de Kalundborg et l'émergence des parcs éco-industriels. Ces modèles de rationalisation de l'écologie industrielle sont des illustrations tangibles du développement durable.

\section{De l'écologie industrielle au développement durable}

L'écologie industrielle n'a été véritablement reconnue et institutionnalisée qu'à la suite du colloque de Washington (mai 1991), parrainé par la National Academy of Science, et de 
la publication dès 1997 d'une revue spécialisée intitulée the Journal of Industrial Ecology (Lifset 1997). Pourtant, dès 1989, certains travaux d'ingénierie précisaient déjà que l'écologie industrielle pouvait être pensée comme une réponse des entreprises à la question du développement durable (Ehrenberg 2004 parle aujourd'hui de «science of sustainability »). Par analogie, le système industriel est associé à un écosystème biologique dont il convient d'analyser les flux de matière (métabolisme industriel) et les prédispositions aux lois énergétiques du monde physique.

\subsection{L'écologie industrielle, une réponse des entreprises à la question du développement durable}

7 La notion "d'écologie industrielle » a été définie en 1989, dans un numéro spécial de la revue «Scientific American " (Pour la Science en français) consacrée à la "gestion de la planète Terre ».Dans un article intitulé « Des stratégies industrielles viables »,Robert Frosch et Nicolas Gallopoulos, tous deux responsables de la Recherche chez General Motors ${ }^{3}$, développent l'idée selon laquelle il devient nécessaire de recycler les biens usagés, d'économiser les ressources et de rechercher des matières premières de remplacement ${ }^{4}$. L'accumulation des déchets et la pollution générée par le progrès technique les conduisent à remettre en cause le modèle de développement des économies industrielles et à formuler la notion d'écosystème industriel: "Ainsi dans un système industriel traditionnel, chaque opération de transformation, indépendamment des autres, consomme des matières premières, fournit des produits que l'on vend et des déchets que l'on stocke. On doit remplacer cette méthode simpliste par un modèle plus intégré: un écosystème industriel » (1989, p. 106). Frosch et Gallopoulos précisent qu'un écosystème industriel pourrait fonctionner comme un écosystème biologique. Les végétaux autotrophes assimilent, par photosynthèse ou chimiosynthèse, des éléments du monde minéral qui se trouvent ainsi intégrés sous une forme réduite dans des molécules organiques; de l'énergie est investie dans ces composés. Les animaux et les végétaux hétérotrophes sont tributaires des précédents et constituent des chaînes trophiques où, de mangeur en mangé, on assiste à un transfert de matière et d'énergie dans le monde vivant. Dans son ouvrage "Vers une écologie industrielle», Suren Erkman (1998, p. 22) souligne que l'article de Frosch et Gallopoulos a joué "le rôle de catalyseur", de sorte que plusieurs interprétations ont donné lieu à un grand nombre de définitions de l'écologie industrielle.Les années 1990 se sont donc cantonnées à stabiliser le vocabulaire d'un courant de pensée en pleine ébullition.

\section{Le champ de l'écologie industrielle}

8 Si les nombreuses interprétations des travaux de Frosch et Gallopoulos n'ont pas permis de déterminer une définition standard de l'écologie industrielle, il semble quela plupart des auteurs (Schulze, Frosch, Risser 1996, Lifset 1998, Ehrenfeld 2004, Erkman 2006) qui se rattachent à ce courant de pensée soient d'accord sur les quatreidées suivantes :

9 i) L'écologie industrielle fait appel en priorité à l'écologie scientifique, aux sciences naturelles (le monde biophysique) et aux sciences de l'ingénieur (Schulze 1996) ${ }^{5}$. Par écologie scientifique, Suren Erkman (1998, p. 10) entend opposer la démarche du chercheur à la contestation politique : «contrairement à la plupart des discussions actuelles sur les questions d'environnement, l'écologie industrielle ne s'aventure pas sur le terrain de 
l'écologisme politique: elle ne fait preuve ni de catastrophisme, ni de son symétrique inverse, l'optimisme technologique à outrance ». L'écologie serait devenue une véritable science suite à la progression de la thermodynamique ${ }^{6}$ des phénomènes irréversibles de Prigogine (1968), à la présentation d'une typologie ${ }^{7}$ des écosystèmes par Eugène Odum (1983) et à l'évocation de lois ${ }^{8}$ "scientifiquement » vérifiées par Kenneth Watt (1973). Les sciences naturelles rappellent qu'un écosystème « is biotic assemblage of plants, animals, and microbes, taken together with their physico-chemical environment » (Kormondv 1969, p. 7). Au sein d'un écosystème, des êtres différents (des producteurs, des consommateurs et des décomposeurs) ont des relations entre eux et avec le milieu minéral (cycles biochimiques). Ces interactions incessantes feront lentement évoluer l'écosystème jusqu'à ce qu'un équilibre stable puisse être atteint entre les divers constituants (Odum 1976). Les sciences de l'ingénieur précisent quant à elles que si le mot écosystème apparait pour la première fois dans les travaux du botaniste A.G Tanley (1935), son émergence est due au succès de différentes théories : la cybernétique de Wiener (1948), la théorie de l'information de Shannon (1949) et l'analyse systémique9 de Forrester (1965). L'art de l'ingénieur consiste « to produce a solution that works in the real world, with all the contraints that task entails. Such contraints may be competitive, ergonomic, regulatory, economic and temporal » (Allenby, Allen, Davidson 2007, p. 8). Il s'agit de résoudre des problèmes environnementaux en mettant en place des procédés industriels optimisés: la transformation des dérivés du pétrole en matériaux polymères, la transformation du minerai de fer en acier, le raffinage des métaux de la famille du platine (Frosch, Gallopoulos [1989]). L'ingénieur accorde une certaine importance au calcul économique. L'Eco-efficiency ${ }^{10}$ est un instrument de l'analyse du développement durable, établissant une relation empirique entre le coût (la valeur) environnemental et l'impact environnemental des activités économiques (Ehrenfeld, 2005).

ii) L'écologie industrielle doit pouvoir mobiliser des disciplines diverses, telles que les sciences économiques, juridiques et sociales. Boons et Roome (2000) insistent sur le fait que l'écologie industrielle étant devenue un phénomène culturel, elle ne peut plus se contenter d'un discours scientifique (l'objectivité du chercheur et de l'ingénieur), elle doit prendre des positions normatives. Ils proposent ainsi « an agenda of research on the cultural and ideological aspects of industrial ecology" (2000, p. 49). De son côté, Isenmann (2002) part du principe que l'écologie industrielle est une philosophie qui prend la nature comme modèle. Il conclut que " $a$ set of arguments drawing on the philosophy of science and on Kantian epistemology and philosophical anthropology is provided to gain greater conceptual clarity and to contribute to laying a solid foundation for industrial ecology's stimulating role in achieving sustainability at large" (2002, p. 27). Enfin, Wells et Orsato (2005) avancent qu'en dépit de ses fondations scientifiques, l'écologie industrielle peine encore à cerner les conditions sociales, environnementales et économiques du développement durable. Un moyen d'enrichir le concept d'écologie industrielle consisterait à se tourner vers les sciences de gestion (la logique de réseaux et la mise en place d'une supply chain posent la question des liens, des relations, des mécanismes de coordination et de la compétitivité ${ }^{11}$ ); les sciences économiques ${ }^{12}$ (les économies d'échelle et la localisation redéfinissent les termes de la concurrence) et les sciences sociales (Wells et Orsato 2005, p. 16) insistent notamment sur le concept de champ organisationnel - «Organizational field ${ }^{13}$ » - contexte dans lequel les organisations sont socialement imbriquées, l'encastrement de Granovetter 2000). 
11 iii) Si un système industriel peut fonctionner comme un écosystème biologique, il ne faut pas prendre cette analogie « au pied de la lettre ». Frosch et Gallopoulos (1989, p. 106) ont ainsi souligné que "l'on ne parviendra jamais à établir un écosystème industriel parfait ${ }^{14}$ ». Ayres (1995) précise qu'il existe des différences importantes entre les organismes biologiques et les unités élémentaires du système industriel, en l'occurrence les entreprises. Si les organismes biologiques sont capables de se reproduire,les firmes ne produisent que des produits ou des services, elles ne peuvent pas produire d'autres entreprises. Par ailleurs, contrairement aux organismes vivants qui évoluent selon un processus temporel long et lent, les entreprises peuvent modifier rapidement leur activité ou/et leurs produits. Levine (2003, p. 33) note une différence fondamentale entre le système biologique et le système industriel : «Products, that is, goods and services exchanged for something of value, are characteristic of industrial systems, but relatively rare in the ecological system ». Ainsi pour reprendre les termes de Suren Erkman (1998, p. 9),on peut décrire le système industriel comme « une certaine configuration de flux et de stocks de matière, d'énergie et d'information, tout comme les systèmes biologiques». Braden Allenby (1994) a exploré le champ de l'écologie industrielle en s'appuyant sur les connaissances propres à l'évolution de la vie sur Terre.La terminologie suggérée par Allenby renvoie à la présence d'écosystèmes de type I, II et III. L'écosystème de type I s'appuie sur un processus linéaire dans lequel les matières premières et les déchets sont illimités. Il n'y aucune activité de recyclage. Les produits industriels sont utilisés de façon frivole, puis rejetés dans l'environnement.Dans l'écosystème de type II, les organismes vivants sont interdépendants et forment des réseaux d'interactions complexes. Cet écosystème est plus efficace que celui du type I, cependant il n'est pas viable à long terme : la diminution des ressources (matières premières) contraste avec l'augmentation inexorable des déchets (Jelinski et al. 1992). L'écosystème de type III a évolué jusqu'à fonctionner de manière entièrement cyclique. Seule l'énergie solaire constitue un intrant. La société industrielle "devrait s'approcher autant que possible d'un écosystème de type III (Erkman 1998, p. 36).

12 iv) Le concept d'écologie industrielle repose sur trois principaux éléments. (1) C'est une vision globale, intégrée, de tous les composants du système industriel et de leurs relations avec la Biosphère. Il s'agit de tirer parti des connaissances sur les mécanismes et le fonctionnement des écosystèmes afin de produire un savoir stratégique (Erkman 1997). Le triptyque (technologie, écologie, économie) apparaît sous la forme d'une quantification des aspects biophysiques (masse, énergie, information) des systèmes de production et de leur interaction avec les systèmes naturels (Koenig, Cantion 1999). (2) La totalité des flux et des stocks de matière, d'énergie et d'informations liés aux activités humaines, constitue le domaine d'études de l'écologie industrielle. Tilley (2003, p. 30) entrevoit l'émergence d'un "eco-system information engineering " capable de créer des systèmes de connaissances et des cycles d'informations susceptibles d'apporter une réponse au management des sociétés industrielles et de services. (3) La dynamique technologique s'avère être un facteur crucial pour favoriser la transition du système industriel actuel vers un système viable ${ }^{15}$ (Ausubel, Sladovich 1989), inspiré par le fonctionnement de l'écosystème biologique. Sur la base d'une étude comparée des fondements (métaphore, approche disciplinaire, position normative, objectifs, perspectives, méthodes et processus) de l'écologie industrielle et de l'innovation, Randles et Berkhout (2006) ont été amenés à supposer l'existence d'une interface entre les deux concepts. La validité et la compatibilité des métaphores (sciences physiques vs sciences sociales); la question des 
échelles (globale, régionale, locale) ; la conceptualisation de la connaissance, de l'information et la compréhension des échecs d'information ; les hypothèses relatives à la théorie de l'agence et au rôle de l'agent ont été étudiées afin de fournir un véritable programme de recherche.

Au-delà de ces spécificités, l'écologie industrielle doit relever un quadruple défi : valoriser les déchets (passer des «bads» aux "goods»); boucler les cycles en minimisant les rejets ; dématérialiser les produits (accroitre la productivité des ressources) et procéder à la décarbonisation de l'énergie (évolution vers un système industriel moins gourmand en énergie fossile).

\section{Ecologie industrielle et métabolisme industriel}

14 Si l'écologie industrielle cherche à s'approcher le plus possible d'un écosystème "viable » à long terme, il devient nécessaire de cerner quantitativement et qualitativement la dimension physique des activités économiques, à savoir les flux et les stocks de matières inhérents à toute activité industrielle (Daniels ${ }^{16}$ 2001, 2002). Cette démarche renvoie à une approche apparue à la fin des années 1980 : l'étude du métabolisme industriel (Ayres 1989). Le concept de "métabolisme » s'applique généralement à une plante ou un animal ${ }^{17}$. Ayres et Simonis $(1995$, p. 1) le définissent comme "the totality of internal processes - both physical and chemical - that supply the energy and nutrients required by an organism as the conditions of life itself'. Ces processus peuvent être décrits en termes de transformations d'inputs (énergie solaire, énergie chimique, eau, air) en biomasse (la substance de l'organisme vivant) et en déchets. Par analogie, le métabolisme industriel ${ }^{18}$ est " l'étude des ensembles des composants biophysiques du système industriel. Cette démarche, essentiellement analytique et descriptive, vise à comprendre la dynamique des flux et des stocks de matière et d'énergie liées aux activités humaines, depuis l'extraction et la production des ressources jusqu'à leur retour inévitable, tôt ou tard, dans les processus biogéochimiques » (Erkman 1998, p. 10). En d'autres termes, c'est tout simplement l'ensemble des transformations physico-chimiques qui permettent de passer des matières premières (biomasse, minéraux, métaux, pétrole) aux biens manufacturés et aux déchets (Lynd, Wang 2004). Les économistes parlent de processus de production. La transformation des biens en services introduit cependant un second terme économique, la consommation. De là, le métabolisme industriel comprend tous les flux de matière et d'énergie qui permettent au système économique de fonctionner, c'est-à-dire de produire et de consommer (Hertwich 2005).

$\mathrm{Vu}$ sous cet angle, le système économique humain occuperait une place à part à l'intérieur du système naturel de la Terre. L'anthroposphère est seulement une partie de la biosphère, elle-même en équilibre dynamique avec le soleil, l'air (l'atmosphère), les océans (l'hydrosphère) et la croûte terrestre (lithosphère). Durant l'ère préindustrielle, l'anthroposphère était plus ou moins en équilibre avec la biosphère et les autres éléments du système terrestre (Husar, 1995). Les humains faisaient partie d'un écosystème naturel, les animaux étaient chassés pour la nourriture et leur peau. Les déchets étaient recyclés par un processus naturel. Même les minerais et les métaux utilisés pour confectionner des armes, des outils ou des pièces, ont été recyclés pendant des millénaires. La révolution industrielle du XVIII ${ }^{m e}$ siècle et l'arrivée des machines à vapeur auraient changé radicalement cette situation. L'exploitation intensive des énergies fossiles et la non prise en compte des contraintes environnementales seraient devenues les moteurs de l'évolution des sociétés humaines. Ayres (1995) précise qu'il existe trois niveaux d'abstraction du concept de métabolisme industriel. A un premier niveau, le métabolisme 
industriel est un ensemble de processus physiques qui transforment les matières premières et l'énergie, plus le travail en produits finis et en déchets. L'offre n'est pas autorégulée. Le contrôle du système est réalisé par la composante humaine.Elle peut exercer un contrôle direct (à travers l'input travail, facteur de production) ou indirect (en tant que consommateur d'output). Le système économique est ainsi stabilisé, au moins dans une structure de marché concurrentielle et décentralisée, lorsque l'offre et la demande de produits (et de travail) sont égalisées selon un mécanisme de prix. De là,le système économique est, par essence, un « metabolic regulatory mechanism" (Ayres 1995, p. 6). A un second niveau, le concept de "métabolisme industriel" renvoie à l'unité élémentaire de l'organisation industrielle, la firme ou l'entreprise manufacturière. Cette dernière est ainsi présentée comme l'unité standard dans la théorie économique. Dès lors, un système économique est " a collection of firms, together with regulatory institutions and worker-consumers, using a common currency and governed by a common political structure" (ibid .). Enfin, à un troisième niveau, le métabolisme industriel focalise son attention sur le cycle de vie des matières et des produits. Or, si la nature est caractérisée par les cycles fermés du carbone, de l'oxygène et de l'hydrogène (Ayres et alii 1996) dans lesquels les processus biologiques jouent un rôle majeur, les firmes font partie d'un système industriel ouvert qui transforme des matières premières et de l'énergie fossile en produits finis et en déchets (Ayres 1999).

La méthodologie du métabolisme industriel consiste donc «à établir des bilans de masse, à estimer les flux et les stocks de matière, à retracer leurs itinéraires et leur dynamique complexes, mais également à préciser leur état physique et chimique » (Erkman 1998, p. 56). Au sein même des entreprises, cette comptabilisation est réalisée sous la formed'une matrice inputoutput (Leontief 1936) et d'une analyse de cycle de vie. Ces "bilans environnementaux " permettent de contrôler les échanges, de connaître le niveau auquel ils se produisent, de savoir comment ils se structurent et comment ils déstructurent l'environnement (Esquissaud 1990). Même si la difficulté de l'étude réside dans la diversité des modalités d'émission des rejets (illustration des pratiques agricoles, Moolenaar 1998, 1999) et la prise en compte des effets de synergie (interactions entre diverses sources de pollution), les bilans environnementaux peuvent s'appuyer sur trois rubriques (Vigneron 1990, p. 18): (1) une forme descriptive de l'activité industrielle incluant les procédés de fabrication et leurs caractéristiques ; (2) une quantification à l'aide d'un inventaire des éléments non monétaires (quantités d'effluents émises, nuisances dans l'air, le sol et l'eau...) et des éléments monétaires (flux de matières premières, quantités produites, investissements, patrimoine bâti et non bâti de l'entreprise...) ; (3) à partir de ces données, une analyse des contraintes législatives, techniques, financières, économiques et commerciales qui expliquent l'attitude de l'entreprise face à la question de l'environnement. Si un nombre suffisant d'entreprises ${ }^{19}$ s'attachent à publier un tel bilan, il sera alors possible d'établir un diagnostic précis de l'état de l'environnement et de surveiller, quantitativement et qualitativement, un élément dont la dégradation est difficilement réversible (Haberl 2001).Le métabolisme industriel pourrait ainsi devenir un outil indispensable pour les responsables politiques, administratifs et économiques, concernés par une gestion optimale des ressources et du territoire, et la planification d'un « développement socio-économique viable » (Erkman 1998, p. 78). Il permettrait non seulement d'optimiser les ressources existantes mais également de détecter des ressources sous utilisées (ou non utilisées) pouvant générer de nouvelles activités. 


\subsection{Ecologie industrielle, thermodynamique et programme bioéconomique}

17 Si l'écologie industrielle et le métabolisme industriel renvoient tous deux à la notion d' "écosystème humain " - c'est-à-dire à un écosystème où le rôle de l'homme est dominant - ce dernier n'échappe pas aux lois énergétiques du monde physique. A la différence des écosystèmes naturels, qui se caractérisent par un flux d'énergie solaire en quantité illimitée et par un recyclage des déchets, les écosystèmes industriels sont traversés par un flux d'énergie fossile provenant d'une source limitée et produisent de façon irréversible des matériaux non recyclés (Vigneron 1990). A ce stade de l'analyse, il convient de revenir sur la littérature scientifique des années 1970, et notamment sur les travaux « précurseurs » de Nicholas Georgescù-Roegen (NGR). Ces travaux se situent au carrefour de la vision thermodynamique du monde, présentée par Sadi Carnot (1824) et des travaux du biologiste Alfred Lotka $(1945,1956)$. La thermodynamique parce qu'elle nous démontre que les ressources naturelles s'épuisent irrévocablement, la biologie parce qu'elle nous révèle la vraie nature du processus économique (Dannequin, Diemer, Vivien 1998).

- La thermodynamique nous enseigne que, dans le processus de production, la quantité d'énergie est conservée (premier principe de la thermodynamique), mais sa forme - et donc sa disponibilité - a changé, de l'énergie libre (ou énergie utilisable) s'est transformée en énergie liée (ou énergie inutilisable), ce que nous appelons le deuxième principe de la thermodynamique, principe dit de Carnot-Clausius, encore appelé loi d'entropie. Pour le dire autrement, le processus économique serait nécessairement entropique ${ }^{20}$, transformant de l'énergie (basse entropie) en déchets et rejets (haute entropie). Entendant pourfendre les références analytiques à la mécanique qui sont monnaie courante en économie, GeorgescùRoegen (1971) a beaucoup fait pour la reconnaissance de la loi d'entropie. Cela l'a amené à intervenir directement dans les controverses scientifiques qui entourent cette loi. Alors que celle-ci concerne habituellement l'énergie, Georgescù-Roegen (1978, p. 361) entend étendre cette loi à la matière, édictant ainsi une quatrième loi de la thermodynamique qui stipule que «dans un système clos, l'entropie de la matière tend continuellement vers un maximum». L'entropie serait donc une loi à laquelle on ne peut échapper, d'où l'insistance de GeorgescùRoegen (1995) sur le caractère irrévocable de cette évolution ${ }^{21}$. Tous les êtres vivants luttent en effet contre l'entropie.

- La biologie souligne que l'homme - comme toutes les espèces naturelles - a toujours utilisé ses organes biologiques afin de puiser la basse entropie de l'environnement. De tels organes propres à chaque espèce vivante sont, selon la terminologie d'Alfred Lotka $(1945,1956)$, les organes endosomatiques. Mais progressivement, les êtres humains se sontdistingués de la plupart des animaux en faisant appel à d'autres instruments qualifiés d'exosomatiques. Avec ces organes détachables, principalement des outils et des équipements techniques « énergétivores ", l'espèce humaine serait parvenue à accomplir de nombreuses réalisations. Les organes exosomatiques seraient même devenus aussi vitaux que les organes endosomatiques (les hommes en sont largement dépendants). Dans ces conditions, le processus économique apparaît bien comme une extension de l'évolution endosomatique, en d'autres termes, comme la continuation de l'évolution biologique (Dannequin, Diemer 1999a). La référence à la biologie permet également de mettre en lumière le concept de biocénose. Ce dernier rappelle que dans les écosystèmes, les différentes espèces d'organismes se rencontrent toujours selon des associations caractéristiques. On peut ainsi étendre ce 
concept aux systèmes industriels en recherchant les meilleures associations (engraisciment ; betterave-biocarburant ; pulpe-papier...)

18 A ce stade de l'analyse, Nicholas Georgescù-Roegen $(1971,1986)$ considèrera que la seule voie pour l'humanité consiste à réorienter son développement exosomatique en intégrant les générations futures (Dannequin, Diemer, 1999b). La prise en compte des générations présentes et futures doit se traduire par la mise en place d'un programme bioéconomique minimal (Georgescù-Rogen 1975, 1978), symbolisant la montée des valeurs sociétales et de l'éthique (Dannequin, Diemer 2004). Tous les espoirs sont ainsi contenus dans la fusion de l'économie et de l'écologie (c'est toutefois l'économie qui devra être absorbée par l'écologie) : «L'un des principaux problèmes écologiques posé à l'humanité est celui des rapports entre la qualité de la vie d'une génération à l'autre et plus particulièrement celui de la répartition de la dot de l'humanité entre toutes les générations. La science économique ne peut même pas songer à traiter ce problème. Son objet, comme cela a souvent été expliqué, est l'administration des ressources rares; mais pour être plus exact, nous devrions ajouter que cette administration ne concerne qu'une seule génération" (NGR 1979, p. 95). Si la« décroissance " prônée par Georgescù-Roegen (1995) paraît difficile à mettre en œuvre (l'homme reste attaché au confort que lui procurent ses organes exosomatiques), le message ne tombera pas dans l'oubli. Frosch et Gallopoulos (1989, p. 114) rappelleront par deux fois qu'un écosystème industriel efficace ne s'établira que "si les réformes de la production s'accompagnent de modifications des habitudes de consommation et des traitements des produits consommés (exemple du tri des ordures bien présent dans des pays comme le Japon, la Suède et la Suisse)». Les industriels et les consommateurs devront changer leurs habitudes s'ils veulent conserver ou améliorer leur niveau de vie, sans souffrir de la dégradation de l'environnement (Tukker, Cohen et al. (2006) insistent sur la notion de "sustainable consumption»). L'écologie industrielle entend s'employer à cette tâche et trouver «des principes et des modèles applicables à la gestion des entreprises et aux processus de production en s'inspirant de l'étude des écosystèmes » (Vivien 2002, p. 5). Cette approche ne se limite pas au stade des idées, elle a pris également forme avec la symbiose de Kalundborg et l'émergence des parcs éco-industriels.

\section{De la symbiose industrielle aux parcs éco- industriels}

Lors de sa conférence à Lausanne, Lifset $(2006$, p. 6) insistait sur ce qu'il a appelé «an initial fascination with Kalundborg». La "symbiose de Kalundborg» illustre la nécessaire interdépendance entre plusieurs processus de production de différentes firmes et le bouclage des flux de matière et d'énergie à mettre en œuvre à l'intérieur d'une zone d'activité industrielle (Vivien 2002). Chertow (2004, p. 2) en donne une définition ${ }^{22}$ explicite : «Industrial symbiosis engages traditionally separate industries in a collective approach to competitive advantage involving physical exchanges of materials, energy, water,and/or by products. The keys to industrial symbiosis are collaboration and the synergistic possibilities offered by geographic proximity". L'exemple de Kalundborg est présenté comme un "écosystème industriel modèle» (Frosch 1995, p. 149). Il a capturé l'attention des économistes (Ehrenfeld, Gertler 1997, Côte, Cohen-Rosenthal 1998, Hardy, Greadel 2002) ; l'imagination des décideurs politiques (Wells, Orsato 2005) et fait de nombreux émules. Les parcs agroindustriels (Labrune 2000, Ozyurt, Realff 2001) ont ainsi été pensés à son image. 


\subsection{La symbiose de Kalundborg}

20 Située au bord de la Mer du Nord, à une centaine de kilomètres de Copenhague, Kalundborg est une petite ville de vingt mille habitants. Kalundborg doit sa fortune principalement à son fjord, l'un des principaux ports accessibles durant l'hiver à cette latitude dans l'hémisphère Nord. C'est précisément l'accessibilité de ce port tout au long de l'année qui se trouve à l'origine du développement industriel de Kalundborg. A partir des années 1950, la ville enregistre l'implantation d'une centrale électrique et d'une raffinerie de pétrole. Au fil du temps, les principales entreprises installées vont échanger des déchets, de la vapeur, de l'eau (à différentes températures et différents degrés de pureté) ainsi que divers produits. A la fin des années 1980, les responsables du développement local réalisèrent qu'ils avaient progressivement et spontanément créé un système qu'ils baptisèrent « symbiose industrielle » (Ehrenberg, Gertler 1997).

\section{Le fonctionnement de l'écosystème}

La symbiose de Kalundborg comprend six partenaires industriels et un acteur public, distants les uns des autres de quelques centaines de mètres seulement, et reliés entre eux par un réseau de pipelines ad hoc (Christensen 2006). La Centrale électrique Asnaes est la plus puissante du Danemark. D'une capacité de 1500 MW, elle est alimentée au mazout puis au charbon (après le $1^{\text {er }}$ choc pétrolier). Elle emploie près de 250 personnes. Statoil est le plus grande raffinerie de pétrole du Danemark. Elle a une capacité supérieure à trois millions de tonnes de pétrole par an et emploie 350 salariés. Novo Nordisk est la plus grande société danoise de biotechnologies, l'un des principaux producteurs d'enzymes industriels et d'insuline. Elle emploie près de 3500 personnes. Gyproc est une société suédoise dont l'usine de Kalundborg produit des panneaux de construction en gypse (14 millions de $\mathrm{m}^{2}$ par an). Elle emploie 165 personnes. Soilrem est une usine de retraitement des sols pollués par les métaux lourds et les hydrocarbures. Elle emploie 65 personnes. Noveren est la propriété de 9 municipalités. Elle retraite près de 126000 tonnes/an de déchets. Enfin, la Municipalité de Kalundborg est un acteur public qui participe activement au projet industriel. Le fonctionnement de l'écosystème Kalundborg peut être présenté de la manière suivante (Nahapétian 2002). L'eau, sous forme liquide ou de vapeur, constitue le déchet valorisé de la manière la plus systématique (Keckler, Allen 1998). Elle provient soit directement du lac Tisso, distant d'une quinzaine de kilomètres, soit du réseau de la municipalité de Kalundborg. La raffinerie de Statoil fournit de l'eau usée pour refroidir la centrale électrique Anaesvaerket. Cette dernière vend de la vapeur à la raffinerie Statoil, à Novo Nordisk (pour ses tours de fermentation), à Gyproc ainsi qu'à la municipalité de Kalundborg pour son réseau de chauffage urbain à distance. La centrale électrique vend même de l'eau chaude à une ferme d'aquaculture qui élève des turbots. 
Fig 1 : La symbiose industrielle de Kalundborg

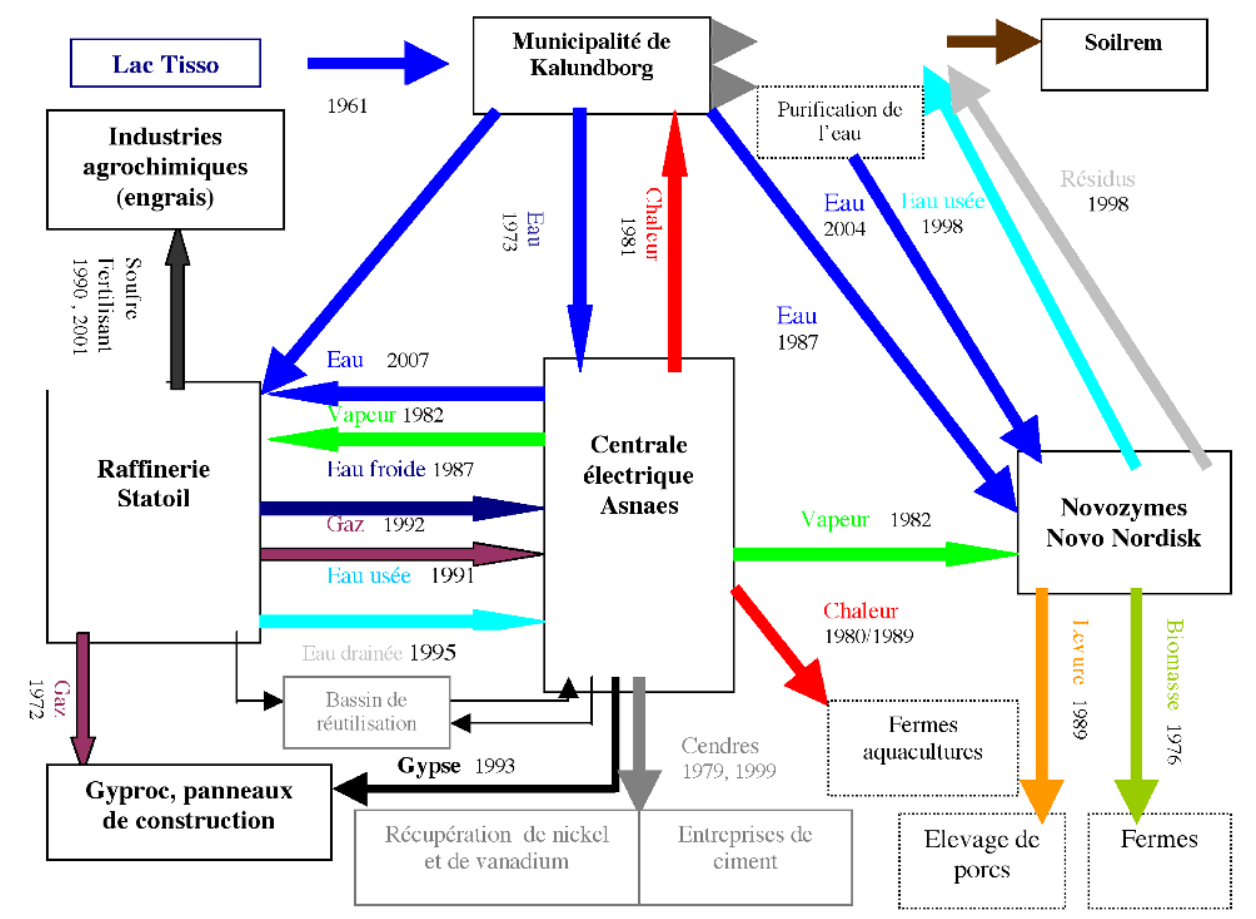

Source : Christensen (2006) certain nombre d'avantages environnementaux et économiques (Erkman 2004). Du point de vue environnemental, l'écosystème industriel faisait apparaître un bilan positif : une réduction de la consommation de ressources (soit $45000 \mathrm{t}$ par an de pétrole, $15000 \mathrm{t}$ par an de charbon et $600000 \mathrm{~m} 3$ par an d'eau, une ressource rare dans la région); une réduction des émissions de gaz à effet de serre et de polluants (soit 175000 tonnes par an de gaz carbonique, 10200 tonnes par an de dioxyde de soufre) et une réutilisation des déchets (130 000 tonnes annuelles de cendres pour la construction routière, 4500 tonnes annuelles de soufre pour la fabrication d'acide sulfurique, 90000 tonnes par an de gypse, 1440 tonnes par an d'azote et 600 tonnes par an de phosphore). Brings Jacobsen (2006) a renouvelé l'opération quelques années plus tard, en cherchant à quantifier les échanges d'eau et de vapeur. Sur la période 1992-2002, le remplacement de l'eau de surface (lac) par de l'eau recyclée concernait près de 1.1 million de m3. Sur la période 1997-2002, les réductions d'émissions de dioxyde de carbone et d'oxyde de nitrogène étaient respectivement estimées à 154000 tonnes et 389 tonnes (il s'agit d'une comparaison de la production de la centrale Asnae avec celle d'une installation au gaz naturel). Du point de vue économique, les investissements nécessaires au bouclage des vingt cinq opérations ont dépassé les 75 millions de dollars. Avec une économie annuelle proche de 15 millions de dollars, le retour sur investissement serait d'environ de quatre à cinq ans pour les projets importants (de deux ans pour les autres).

Au-delà de ses nombreux avantages, lesystème de Kalundborg ne doit pas être "idéalisé " (Erkman 2004, p. 4) mais plutôt servir d'exemple. Deux inconvénients ${ }^{23}$, ont ainsi été formulés par certains observateurs (Sterr, Ott 2004) : une certaine rigidité des échanges et un risque systémique en cas de défection de l'un des coopérants. 


\section{Les enseignements de Kalundborg} processus spontané, qui s'est progressivement (de 1961 à 2007) mis en place sur des bases commerciales qui satisfont toutes les entreprises (scénario win-win). Suren Erkman note que « les échanges obéissent aux lois du marché » 1998, p. 26). Chaque livraison de "déchets " entre les partenaires fait l'objet d'une négociation séparée et confidentielle. La symbiose industrielle de Kalundborg apparaît sous la forme d'un « réseau environnemental » de plus de 20 accords commerciaux bilatéraux entre 6 firmes et une municipalité. Trois types de projets ont vu le jour : le recyclage de l'eau (12 projets), l'échange d'énergie (6 projets) et le retraitement des déchets (7 projets). Depuis 1996, le «Symbiosis Institute» constitue la mémoire vivante de cette réussite industrielle. Ensuite, le succès du système repose sur une bonne communication ${ }^{24}$ entre les partenaires. Christensen (2006, p. 47-48) met en avant trois facteurs clés : la confiance mutuelle "Participants must fit, but be different ", la proximité géographique "There has to be a short physical distance between the participants ", le style de management (partage de certaines valeurs), "There has to be a short mental distance between the participants ». Enfin, pour devenir opérationnel, ce système doit être intégré dans l'organisation structurelle des entreprises. Dans le domaine du management, l'écologie industrielleentraîne des conséquences majeures. D'une part, elle remet en cause la focalisation de l'entreprise sur le produit. En effet, il s'agit de donner autant d'importance à la valorisation des déchets. D'autre part, les entreprises doivent établir une sorte de Management "over the fence », c'est-à-dire une chaîne collaborative pour assurer une gestion optimale des ressources (Esty, Porter, 1998). Le fait d'optimiser tous les flux de matière et d'énergie mobilisés par les entreprises (de la matière première jusqu'au produit fini) se traduit «tôt ou tard par une performance et une compétitivité accrue " (Erkman, 1998, p. 33). Pour améliorer l'efficacité d'un tel système, il sera cependant nécessaire de favoriser certains panachages industriels (ceci renvoie aux biocénoses industrielles) propices aux échanges de déchets et de ressources (Brings, Jacobsen, Anderberg 2004).

\subsection{Elaboration d'un parc agro-industriel}

A la suite du succès de Kalundborg, de nombreux travaux (Chertow 2007, Zhu and al. 2007, Beers et alii 2007) ont cherché à cerner la nature et les caractéristiques de la symbiose afin de la reproduire ${ }^{25}$ ailleurs. Une série d'initiatives et de projets ont vu le jour. Le concept de «parc éco-industriel ${ }^{26}$ » a été conçu dans les années 90 . Il s'agit d'une zone dans laquelle les entreprises coopèrent pour optimiser systématiquement l'usage des ressources et la valorisation des déchets (Chertow 1999, Peck 2001). L'échelle appropriée du parc dépend du contexte économique, politique et culturel du territoire (Brullot 2006) mais également des effets de synergie (une entreprise, située à une grande distance du complexe industriel, peut être intégrée au parc, si elle est la seule à maîtriser une technologie ou à recycler un déchet dangereux). Suren Erkman (2004, p. 5) préfère parler de « réseaux éco-industriels » (dont les parcs représenteraient un cas particulier) toutefois on pourrait également rapprocher les parcs du concept de "Districts industriels ", une forme d'organisation industrielle et territoriale, chère à Marshall (1919) et à Becattini $(2003,2004)$. Afin d'illustrer ces propos, nous présenterons, dans ce qui suit, ce qu'il serait convenu d'appeler un projet de district agro-industriel qui a été soutenu par L'ADEME, l'

Développement durable et territoires, Varia (2004-2010) | 2007 
Institut Supérieur Agricole de Beauvais (ISAB) et la Vannerie Candas (Somme). A l'image des travaux de Ozyurt et Realff $(1998$, p. 13), on peut avancer que l'émergence de cet écosystème industriel passe par trois phases : « (1) locating sources and sinks of potential materials for exchange or upgrading;(2) filtering feasible exchanges, the exchange candidates, and locations for new infrastructure; and (3) generating optimal configurations for the industrial ecosystem based on the objective of maximizing the system benefit, defined in the particular case study as the revenue that is generated by the system".

Les saules (utilisés pour la culture de l'osier) ou Taillis à Très Courte Rotation (TTCR) représentent à la fois une nouvelle pratique agricole (qui s'apparente à la sylviculture) et une illustration de l'élaboration d'un parc agro-industriel (Arronsson 1995). Cette culture consiste à planter une grande quantité de boutures de saules qui se développeront en souches. Ces souches permettront d'obtenir un nombre considérable de branches de saules qui peuvent être coupées et broyées. Les TTCR présentent deux particularités (Rosenqvist, Aronsson, Hasselgren, Perttun 1997). D'une part, ils peuvent approvisionner des chaufferies à bois ou des gazogènes qui produisent de la chaleur ou de l'électricité respectivement. Cette pratique, fortement développée dans les pays scandinaves, permet ainsi de transformer la biomasse en une énergie locale, renouvelable et respectueuse de l'environnement car elle ne participe pas à l'effet de serre. D'autre part, ils peuvent absorber les éléments nutritifs et l'eau contenus dans les rejets liquides des stations d'épuration ou des entreprises agro-alimentaires (eaux usées et boues). Ces effluents, qui constituent une menace pour l'environnement (pollution des milieux aquatiques, contamination des cultures alimentaires par des boues urbaines...) sont valorisés grâce aux saules en amendements organiques (voir également le Kageröd Recycling Projet de la Municipalité de Svalov ${ }^{27}$, Suède). Les TTCR constituent avec le sol un biofiltre végétal qui absorbe l'azote et le phosphore présents en forte quantité dans ces effluents (Ahman, Backlund, Larsson [1999]). Les saules peuvent ainsi constituer la clé de voûte d'un véritable parc Agro-Industriel (c'est à dire qu'ils peuvent faire le lien entre le traitement des effluents et la production d'énergie pour des entreprises ou des collectivités) au même titre que l'expérience de Kalundborg.

\section{Intrants et équipements nécessaires pour le Taillis à Très Courte Rotation}

Les intrants d'une telle culture sont minimes par rapport aux grandes cultures. Ceux-ci sont principalement la lumière, l'eau et des éléments nutritifs : azote, phosphore et potassium. Pour éviter le développement des mauvaises herbes, on utilise des herbicides ou on travaille superficiellement la terre avec une herse. Pour éviter le développement des maladies, on choisit des boutures sélectionnées génétiquement et on mélange dans la parcelle plusieurs clones afin d'éviter des contaminations trop rapides du TTCR (insectes, parasites...). La culture du TTCR demande du temps principalement lors de la phase de démarrage (bonne préparation $\mathrm{du}$ sol, plantation des boutures...). Ensuite, la récolte du bois nécessite du matériel spécifique pour couper et broyer les tiges de saules. Le séchage du bois, si nécessaire, représente aussi une dépense d'énergie dans le schéma d'obtention de bois sec en tant que combustible. La figure 2 présente les divers intrants et équipements nécessaires pour assurer l'implantation et le développement des saules en TTCR.

Un tel système présente des avantages et des inconvénients techniques, environnementaux et économiques. Du point de vue technique, les TTCR ont une grande 
capacité d'adaptation sur de nombreux sols. Il est également possible de valoriser des friches agricoles et industrielles. Peu d'interventions sont nécessaires sur le terrain. Par contre, les TTCR exigent l'utilisation de machines spécifiques qu'il faut importer d'Europe du Nord. Le développement des mauvaises herbes lors de la phase d'implantation peut être préjudiciable à la productivité des saules. Il est enfin nécessaire d'avoir une structure pour le séchage du bois (abri aéré pour les tiges, silo de stockage, séchage pour les plaquettes). Du point de vue environnemental, les TTCR exigent peu d'intrants chimiques par rapport aux grandes cultures. Ils limitent les phénomènes de lessivage en régions de grandes cultures (zone tampon), augmentent la biodiversité du milieu (faune et flore), ont un impact positif ${ }^{28}$ sur le paysage et un effet coupe vent (réduisant l'érosion éolienne). Du point de vue économique, le TTCR fournit des revenus proches des grandes cultures avec moins de travail. Une telle diversification entraîne cependant des problèmes de trésorerie (les retours sur investissement ne surviennent qu'au bout de la $4^{\text {ème }}$ année). Par ailleurs, la rentabilité de cette culture passe actuellement par une subvention à l'implantation et des primes à la jachère.

Fig 2 : Intrants et matériels nécessaires à la culture du TTCR de saules

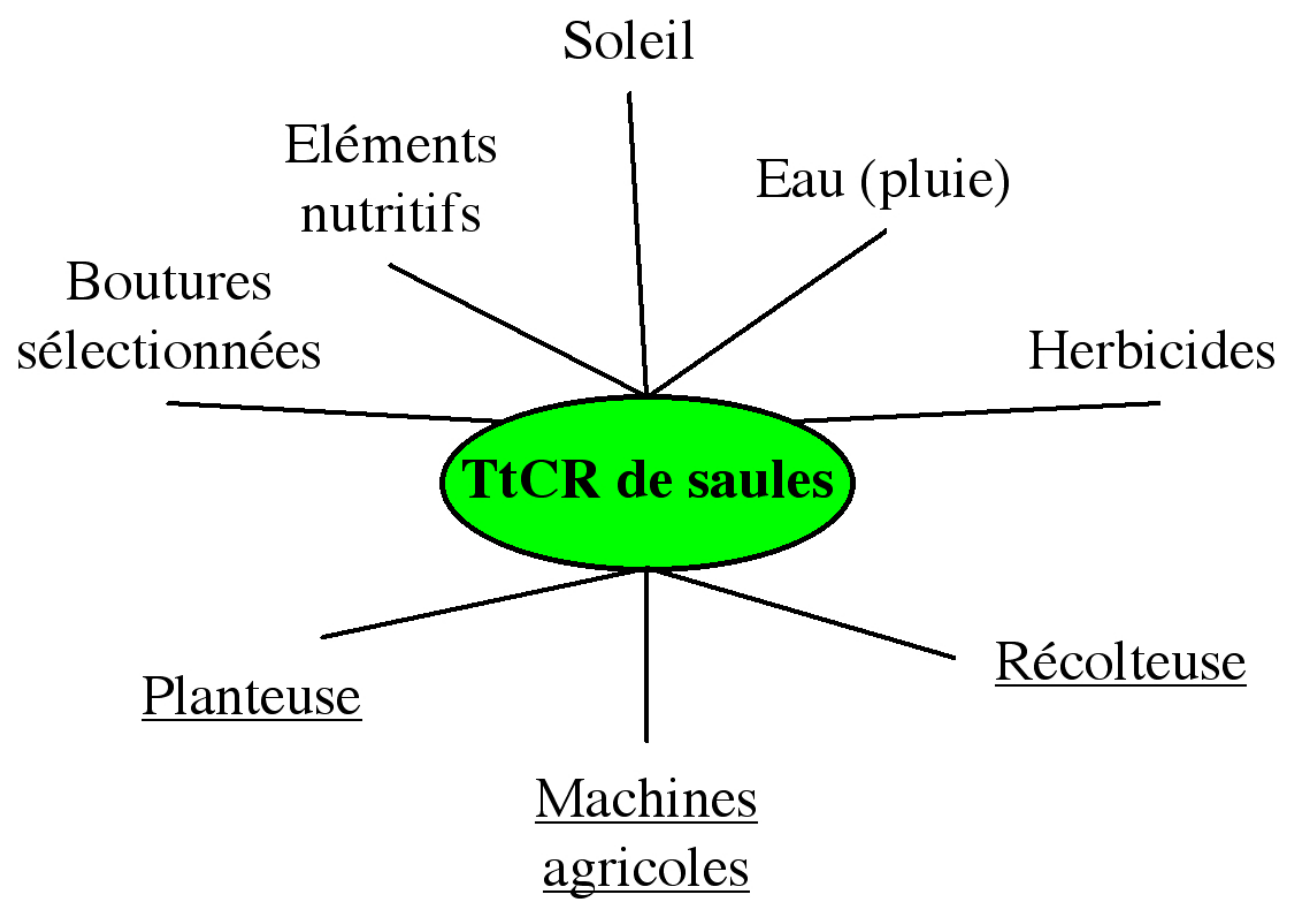

Source : Labrune (2000)

\section{La production de chaleur ou d'électricité}

Les plaquettes de saules sont stockées jusqu'à ce qu'elles aient atteint un taux d'humidité de l'ordre de $15-25 \%$. Cette étape nécessite un lieu de stockage pour les plaquettes mais peut se faire à l'extérieur pour les saules en tiges. Les plaquettes de saules sont ensuite utilisables en tant que combustible pour des chaufferies ou des gazogènes ${ }^{29}$. L'autre valorisation possible de ces plaquettes consiste à transformer le bois en gaz qui génère de l'électricité. Cette électricité peut alors servir aussi bien pour le chauffage ${ }^{30}$ que pour la consommation électrique de l'usager (fig. 3). 
Fig 3 : De la culture de saules à la production d'énergie

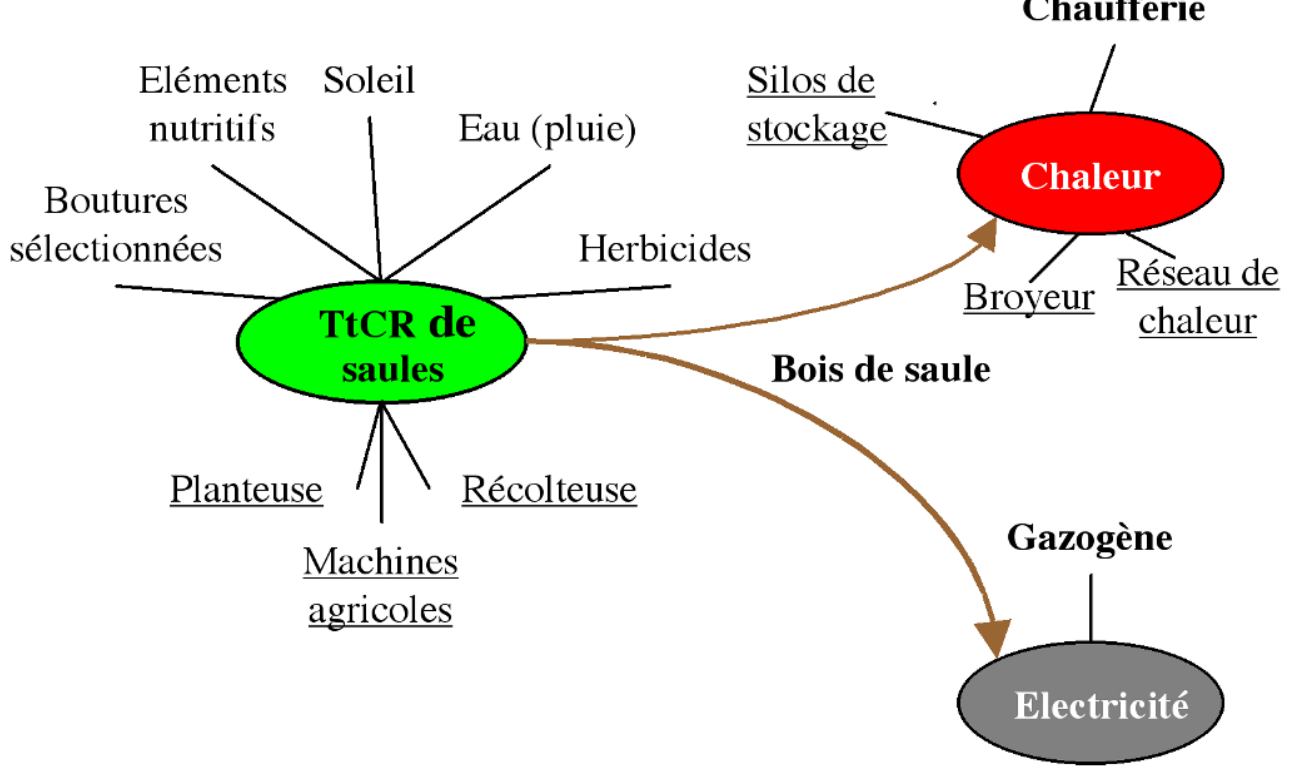

Source : Labrune (2000)

De la même manière que les intrants, on peut noter que la production d'énergie à partir des TTCR génère une série d'avantages et d'inconvénients du point de vue technique, environnemental et économique. Concernant la partie technique, il convient de souligner que l'approvisionnement de la chaufferie peut être complété par les déchets et sousproduits du bois de la région. Par ailleurs, les chaufferies semi-automatiques assurent l'alimentation de la chaudière en fonction des besoins de l'usager. La manipulation du bois de saules du taillis à la chaufferie est cependant lourde. Les petites unités peuvent avoir des problèmes de bourrage si le bois utilisé est trop humide. Sur la partie environnementale, il faut insister sur le fait que les saules fixent plus de $\mathrm{CO}^{2}$ lors de leur croissance qu'ils n'en dégagent lors de la phase de combustion. En outre, la combustion du bois, contrairement au fuel et au charbon, ne dégage pratiquement pas de soufre. Concernant les dimensions économiques et sociales, le faible coût de fonctionnement d'une chaufferie à bois permet un retour sur investissement de 3,5 ans(par rapport au gaz) à 7 ans (par rapport au fuel) ${ }^{31}$. L'ADEME subventionne les projets de chaufferies à bois à hauteur de $30 \%$ du montant de l'investissement. Enfin, pour un emploi induit par une chaufferie fuel ou gaz, la filière bois est susceptible d'induire 3 à 4 emplois. Un projet local de chauffage au bois peut ainsi être à l'origine d'un développement local. Toutefois, les engagements de l'Etat dans les énergies renouvelables telles que la biomasse ou le solaire restent encore timides ${ }^{32}$ (et ce malgré les mesures gouvernementales prises en 2006). Par ailleurs, le contexte juridique et fiscal ne favorise pas pour l'instant l'utilisation du bois-énergie.

\section{Le recyclage des eaux usées et des boues résiduelles}

31 Les stations d'épuration réalisent le traitement primaire et secondaire des eaux usées collectées dans les zones d'assainissements collectifs (fig. 4). Au cours de ces traitements, les boues résiduelles constituent un déchet qu'on peut valoriser en les épandant sur les TTCR de saules juste après le recépage ${ }^{33}$. On peut également irriguer les saules avec les 
eaux usées pré-épurées, ce qui permet d'assurer la dénitrification et la déphosphatation avant le rejet dans le milieu naturel, tout en créant de la matière première : le bois. Par ailleurs, les saules permettent de valoriser les effluents et les boues issus des entreprises agro-alimentaires. Les éléments nutritifs contenus dans ces rejets liquides sont ainsi recyclés en biomasse ${ }^{34}$. Dans ce cas, les boues proviennent de la station d'épuration de l'entreprise dont les effluents ne peuvent être épandus directement sur les TTCR.

Fig 4 : Flux de matières et équipements d'un parc agro-industriel de saules

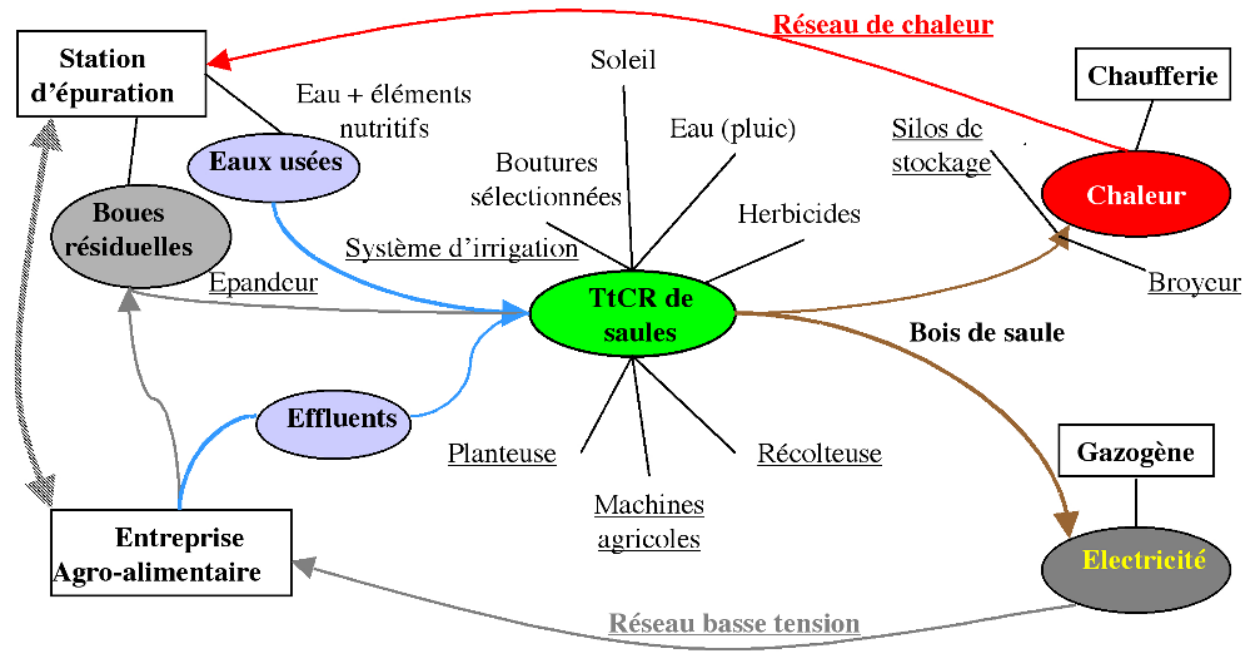

Source : Labrune (2000)

Du point de vue technique, un tel système présente certains avantages. Les entreprises agro-alimentaires peuvent traiter leurs effluents avec peu de structures à entretenir (par rapport à uns station d'épuration). Le traitement tertiaire des eaux usées grâce aux TTCR est aussi efficace que les techniques physico-chimiques. L'épandage des boues sur les saules peut se faire à des moments où d'autres cultures ne le permettent pas. Il existe cependant des inconvénients. Les TTCR exigent beaucoup de place. Ce système ne convient que pour des effluents et des eaux usées peu chargés en pollution. Les bassins de stockage des eaux usées et l'épandage des boues peuvent engendrer des mauvaises odeurs. Le système de contrôle des eaux usées représente une contrainte réglementaire qu'il faut prendre en compte pour les petits projets d'irrigation. Du côté environnemental, grâce à leur pouvoir épurateur, les saules permettent de traiter les eaux usées au niveau tertiaire et empêchent les phénomènes d'eutrophisation des milieux aquatiques. L'épandage des boues sur une culture non-alimentaire permet d'éviter le "recyclage de la pollution". Les boues et eaux usées appliquées d'une manière excessive peuvent cependant engendrer des phénomènes de lessivage. Concernant le volet économique et social, la valorisation de ces déchets en biomasse permet un retour sur investissement rapide des installations de traitement et peut même créer de la richesse pour l'entreprise. Les entreprises agro-alimentaires bénéficient avec une telle technologie d'une image de marque environnementale: c'est le "marketing vert". Les saules seraient ainsi une réponse au problème de traitement des boues de stations d'épuration dans un contexte agricole de plus en plus défavorable. Il convient toutefois de noter que les investissements de départ (achat ou location des terrains notamment) peuvent constituer un facteur limitant au traitement tertiaire des eaux usées via les 
saules. De plus, le choix d'une telle technologie peut sembler inutile pour les petites et moyennes stations d'épuration qui ne sont pas obligées pour l'instant de réaliser un traitement tertiaire de leurs eaux usées. Enfin, les Industries agro-alimentaires risquent de rencontrer des difficultés pour louer ou acheter des terrains destinés à traiter leurs effluents.

\section{Conclusion}

En l'espace de deux décennies, l'écologie industrielle s'est construite autour d'un corpus théorique associant les sciences de l'ingénieur, les sciences de la terre (thermodynamique et biologie), les sciences sociales, économiques et juridiques. L'écologie serait ainsi devenue une véritable science (avec ses propres lois et son propre langage), et l'entreprise, un vecteur du développement durable. Par analogie, le système industriel est associé à un écosystème biologique dont les principaux objectifs sont la valorisation systématique des déchets, la minimisation des pertes par dissipation (création de réseaux d'utilisation des ressources et des déchets), la dématérialisation de l'économie (optimisation de l'utilisation de la matière) et la décarbonisation de l'énergie.L'écologie industrielle analyse ainsi «local, regional, and global uses and flows of materials and energy in products, processes, industrial sectors, and economies. It focuses on the potential role of industry in reducing environnemental burdens throughout the product life cycle from the extraction of raw materials, to the production of goods, to the use of those goods and to the management of the resulting wastes" (Lifset, 2006, p. 2).L'étude du métabolisme industriel (démarche consistant à comprendre la dynamique des flux et des stocks de matière et d'énergie liées aux activités humaines) constitue un préalable indispensable à l'écologie industrielle. L'écosystème industriel fait apparaître deux niveaux d'abstraction: 1 / un processus de production combiné à un niveau de consommation dans le cadre d'une structure de marché concurrentielle régulée par les prix ; 2/ une entité élémentaire, l'entreprise, qui doit comptabiliser ses flux d'énergie sous la forme de bilans matière (on parle également de bilan environnemental). Sans prôner la décroissance (Georgescù-Roegen, 1995), les tenants de l'écologie industrielle recommandent aux industriels de procéder à un ensemble d'opérations de rationalisation de la production. Des idées qui, sur le terrain, seront illustrées par la symbiose industrielle de Kalundborg et l'émergence des parcs écoindustriels.Dans cette nouvelle approche de l'écologie, le recyclage des déchets ne serait plus une fin en soi. Le développement durable doit désormais mener à une valorisation collective et coordonnée des déchets (passage de la valeur d'échange à la valeur d'utilisation). Et c'est de cette manière que les sciences de l'ingénieur pourront être pensées comme des alternatives « technologiques » au service des Société Humaines.

\section{BIBLIOGRAPHIE}

ACDE (1998), Rapport annuel de l'Association pour le Développement des Cultures Energétiques, Lille. 
Ahman I., Backlund A., Larsson S. (1999), Willow Wastewater News, n³, Svalöv, (Sweden), European Commission DG IV, Agriculture, 6 p.

Allenby B.R, Allen D.T, Davidson C.I (2007), “Teaching sustainable engineering”, Journal of Industrial Ecology, vol 11, $\mathrm{n}^{\circ} 1$, p. 8-10.

Aronsson P. (1995), Energy Forestry and Nature Preservation, Uppsala Swedish University of Agriculture Sciences, $15 \mathrm{p}$.

Ayres R.U, Ayres L.W (1999), “The Life Cycle of Chlorine , Part IV : Accounting for Persistent Cycllic Organo-Chlorines”, Journal of Industrial Ecology, vol 3, n²-3, p. 121-159.

Ayres R.U, Ayres L.W (1996), «Industrial Ecology: Towards Closing the Materials Cycle » Edward Elgar. Ayres R.U, Simonis U.E (1995), Industrial Metabolism : Restructuring for Sustainable Development, United Nations Publication, New York.

Ayres R.U (1989), «Industrial Metabolism and Global Change », International Social Science Journal, pp. 363 - 373. Traduction française, « Le métabolisme industriel et les changements de l'environnement planétaire ", Revue internationale des sciences sociales, n²121, p. 401-412.

Ausubel J.H, Sladovich H.E (1989), Technology and Environment, National Academy Press, Washington.

Becattini G. (2004), Industrial Districts : A New Approach to Industrial Change, Edward Elgar Publishing Williston, Vermont.

Becattini (2001), The Caterpillar and the Butterfly: An Exemplary Case of Development in the Italy of the Industrial Districts, Felice Le Monnier, Firenze.

Becattini G., Bellandi M., Ottati G.D, Sforzi F. (2003), From Industrial Districts to local Development: An Itinerary of Research, Edward Elgar Publishing, Cheltenham.

Beers V.D, Corder G., Bossilkov A., Berkel V.R (2007), "Industrial Symbiosis in the Australian Minerals Industry”, Journal of Industrial Ecology, vol 11, n²1, p. 55-72.

Bourg D. (1996), Les scénarios de l'écologie, Paris, Hachette.

Bourg D. (2002), Le développement durable a-t-il un avenir ?, Le Pommier.

Bourg D. (2003), Le nouvel âge de l'écologie, Descartes et Cie.

Bourg D., Erkman S. (2003), Perspectives on industrial Ecology, Sheffield, Greenleaf Publishing.

Bourg D., Rayssac G.L (2006), Le développement durable, Gallimard.

Brings Jacobsen N. (2006), "Industrial Symbiosis in Kalundborg”, Journal of Industrial Ecology, vol $10, \mathrm{n}^{\circ} 1-2$, p. 239-255.

Brings Jacobsen N., Anderberg S. (2004), "Understanding the Evolution of Industrial Symbiotic Networks - The Case of Kalundborg" in Economics of Industrial Ecology: Materials, Structural Change and spatial scales, Van Der Bergh J., Janssen M. (ed), Cambridge, MA: MIT Press.

Brown L. (1991), «Le défi planétaire » Sang de la Terre.

Brunel S. (2004), Le développement durable, PUF.

Brullot S. (2006), « L'écologie industrielle : mise en œuvre à l'échelle d'un territoire », Scientific Workshop 'Frontiers of Research in Industrial Ecology', University of Lausanne, Nov 27 - Dec 1th, 16 p. Carnot S. (1824), « Réflexions sur la puissance motrice du feu et sur les machines propres à développer cette puissance » Paris, Bachelier. Réédition en 1872, 1878, 1913, 1953. 
Chertow M.R (2007), “Uncovering Industrial Symbiosis”, Journal of Industrial Ecology, vol 11, n¹, p. 11-30.

Chertow M.R (2004), "Industrial Symbiosis" in Encyclopedia of Energy, Cleveland C.J (ed), Oxford.

Chertow M.R (1999), « The Eco-industrial Park Model Reconsidered », Journal of Industrial Ecology, vol 2, n³, p. 8-10.

Côte R., Cohen-Rosenthal E. (1998), “Designing Eco-industrial parks: A synthesis of some experiences”, Journal of Cleaner Production, vol 6, p. 181-188.

Christensen J. (2006), “The History of the Industrial Symbiosis at Kalundborg, Danemark”, Scientific Workshop 'Frontiers of Research in Industrial Ecology', University of Lausanne, November 27 - December 1 th, $49 \mathrm{p}$.

Daniels P.L, Moore S. (2001), “Approaches for Quantifying the Metabolism of Physical Economies : Part I : Methodological Overview”, Journal of Industrial Ecology, vol 5, n4, p. 69-93.

Daniels P.L (2002), “Approaches for Quantifying the Metabolism of Physical Economies: A Comparative Survey: Part II: Review of Individual Approaches”, Journal of Industrial Ecology, vol 6, $\mathrm{n}^{\circ} 1$, p. 65-88.

Dannequin F., Diemer A. (2004), «Nicholas Georgescù-Roegen : vers la décroissance? » texte disponible sur le site http://www.oeconomia.net, rubrique Histoire de la pensée économique, 4p.

Dannequin F., Diemer A., Petit R., Vivien F-D (2000), « la nature comme modèle ? Ecologie industrielle et développement durable ", Cahiers du CERAS, n 38, mai, p. 62-75.

Dannequin F., Diemer A. (1999a), « La place de la biologie et de la thermodynamique dans la théorie contemporaine : l'oeuvre scientifique de Nicholas Georgescù-Roegen », Colloque de l'ACGEPE, 26-27 septembre, Paris, 8 p.

Dannequin .F, Diemer .A (1999b), « De l'entropie à la constitution d'un programme bioéconomique : Le grand projet de Nicholas Georgescù-Roegen ", Cahiers du CERAS, Décembre, $\mathrm{n}^{\circ}$ 42, pp. 1- 9 .

Dannequin .F, Diemer .A, Vivien F.D (1999a), « Ecologie industrielle et développement durable » Colloque de Reims, Hermès, 8 p.

Dannequin .F, Diemer .A, Vivien F.D (1999b), «Industrielle ou politique? Quelle écologie pour le développement durable ?» International Conference on Industrial Ecology and Sustainability, Université technologique de Troyes les 22-25 septembre, 8 p.

Dannequin F., Diemer A., Vivien F.D (1998), «Thermodynamique, biologie et économie chez GeorgescùRoegen » Colloque de Reims, journées Hermès,7 décembre, 8 p.

Diemer A. (2005), « Le Développement Durable vu par les économistes », Journées « Culture, Economie et Développement durable », IUFM Auvergne, 13 p.

Ehrenfeld J.R, Gertler N.(1997), "Industrial Ecology in Practice : The evolution of interdependence at Kalundborg”, Journal of industrial Ecology, winter, vol 1, n¹, p. 67-79.

Ehrenfeld J.R (2004), “Can Industrial Ecology be the ‘Science of sustainability’?, Journal of Industrial Ecology, vol 8, n¹-2, p. 1-3.

Ehrenfeld J., Chertow M.R (2004), "Industrial Symbiosis: The Legacy of Kalundborg” in Handbook of Industrial Ecology, Ayres R. (ed), Northampton, UK: Edward Elgar.

Ehrenfeld J.R (2005), « Eco-efficiency : Philosophy, Theory and Tools », Journal of Industrial Ecology, vol $9, n^{\circ} 4$, p. $6-8$. 
Erkman S. (1994), «Ecologie industrielle, métabolisme industriel et société d"utilisation » Genève, ICAST.

Erkman S. (1997), «Industrial Ecology : An Historical View » Journal of Cleaner Production, vol 5, $\mathrm{n}^{\circ}$ $1-2$, p. $1-10$.

Erkman S. (1998), «Vers une écologie industrielle : comment mettre en pratique le développement durable dans une société hyper-industrielle » Editions Charles Leopold Mayer, réédition, 2006.

Erkman S. (2001), «L'écologie industrielle, une stratégie de développement » Le débat, n¹13, p.106-121.

Erkman S. (2004), «L'écologie industrielle, une stratégie de développement », Exposé, Bruxelles, 9 p.

Esquissaud P. (1997), «Ecologie Industrielle » Paris, Hermann.

Esty D.C, Porter M.E (1998), «Industrial Ecology and Competitiveness : Strategic Implications for the Firm ", Journal of Industrial Ecology, vol 2, n²1, p. 35-43.

Fisher-Kowalski M. (1998), “Society's Metabolism: The intellectual History of Materials Flow Analysis, Part I, 1970-1998”, Journal of Industrial Ecology, vol 2, n¹, p. 61-78.

Fisher-Kowalski M., Hüttler W. (1998), “Society’s Metabolism: The intellectual History of Materials Flow Analysis, Part II, 1970-1998”, Journal of Industrial Ecology, vol 2, n4, p. 107-136.

Frosch R. (1995), « L'écologie industrielle du XXe siècle », Pour la science, 217, p. 148-151.

Frosh R.A (1992), «Industrial Ecology : A philosophical Introduction » Proceedings of the National Academy of Sciences of the USA, vol 89, p. $800-803$.

Frosch R.A, Gallopoulos N.E (1992), « Towards an Industrial Ecology », p. 269 - 292, in Bradshow A.D, «The Treatment and Handling of Wastes » Chapman and Hall, London.

Frosch R.A, Gallopoulos N.E (1989), «Strategies for Manufacturing », Scientific American, vol 261, Special Issue «Managing Planet Earth », September, p. 144 - 152). Traduction française «Des stratégies industrielles viables », Pour la Science, n¹45, novembre 1989, p. 106 - 115.

Georgescu-Roegen N. (1995), «Demain, la décroissance: entropologie-écologie-économie», Sang de la Terre.

Georgescù-Roegen N. (1987), «Entropy» in J. Eatwell, M. Milgate, P.K Newman, The New Palgrave, A Dictionary of Economics, vol I, London, Mc Millan Press, (p 153 - 156)

Georgescu-Roegen N. (1986), "Man and production”, in Baranzani M. et Scazzieri R. (eds) " Foundations of economics", Basil Blackwell.

Georgescu-Roegen N. (1979), "Methods in economic science", Journal of economic issues, vol XIII, nº 2.

Georgescù-Roegen Nicholas (1978) «De la Science Economique à la Bioéconomie », Revue d'Economie Politique,t LXXXVIII, n 3, Mai-Juin,p. 337 - 382.

Georgescu-Roegen N. (1978), "Mechanistic dogma and economics", British review of economic issues, 2.

Georgescù-Roegen N. (1977), « What thermodynamics and Biology Can Teach Economists », Atlantic Journal Economic, vol 5, p. 13 - 21.

Georgescù-Roegen (1977 a), «What Thermodynamics and Biology Can Teach Economists », BioScience vol XXVII, avril, p. 266 - 270.

Georgescu-Roegen .N (1977 b), «Inequality, Limits and Growth from a Bioeconomics Viewpoint » Review of Social Economy, vol XXXV, p. 361 - 375. 
Georgescù-Roegen N. (1976), Energy and Economic Myths, New York, Pergamon Press.

Georgescù-Roegen N. (1975), « Bio-Economic Aspects of Entropy » dans «Entropy and Information in Science and Philosophy » J. Zeman, Amsterdam Elsevier.

Georgescu-Roegen N. (1971), «The Entropy Law and the Economic Process » Cambridge , Harvard University Press.(4ème édition 1981).

Gorz A. (1978), Ecologie et politique, Paris, Ed. du Seuil.

Gorz A. (1988) , Métamorphoses du travail. Quête de sens, Paris, Galilée.

Graedel T.E, Allenby B.R (1995), «Industrial Ecology » Englewood Cliffs, NJ, Prentice Hall

Graedel T.E. (1996), « On the Concept of Industrial Ecology », Annu. Rev. Energy Environ., 21, pp.

69-98.

Granovetter M. (2000), Le marché autrement, Desclée de Brouwer, Paris, 239 p.

Grinevald J. (1996), « Nicholas Georgescù-Roegen : La Ley de la Entropia y el Proceso Economico » Madrid, Fundacion Argentaria/ Visor 1, p. 1 - 37.

Grinevald J. (1993), "Georgescu-Roegen-Roegen : bioéconomie et biosphère" ;Silence n¹64, avril.

Grinevald J. (1990), «L'effet de serre de la biosphère : de la révolution thermo-industrielle à

l'écologie globale ", Stratégies énergétiques, Biosphère et Société, Genève, vol 1, p. 9 - 34.

Grinevald J. (1973), «La notion d'entropie dans la pensée contemporaine, fragments pour une recherche holistique sur le temps, le désordre et la mort », Mémoire de maîtrise, Faculté de philosophie, Besançon.

Haberl H. (2001), “The Energetic Metabolism of societies Part I: Accounting Concepts”, Journal of Industrial Ecology, vol 5, $\mathrm{n}^{\circ} 1, \mathrm{p} .11-33$.

Haberl H. (2001), “The Energetic Metabolism of societies Part II: Empirical Examples”, Journal of Industrial Ecology, vol 5, n², p. 71-88.

Hardy C., Greadel T. (2002), "Industrial Ecosystems as food webs”, Journal of Industrial Ecology, vol $6, \mathrm{n}^{\circ} 1$, p. 29-38.

Hertwich E.G (2005), “Consumption and Industrial Ecology”, Journal of Industrial Ecology, vol 9, n², p. $1-6$.

Huppes G., Ishikawa M. (2005), « Why Eco-Efficiency? », Journal of Ecology Industrial, vol 9, n4, p. $2-5$.

Huppes G., Ishikawa M. (2005), « A Framework for quantified Eco-Efficiency Analysis », Journal of Ecology Industrial, vol 9, n4, p. 25-41.

Husar H.B (1995), “Ecosystem and the Biopshere : Metaphors for human incluced material flows", in Ayres R.U, Simonis U.E (1995), Industrial Metabolism : Restructuring for Sustainable Development, United Nations Publication, New York.

Illich I. (1973), La convivialité, trad. fse, Paris, Ed. du Seuil.

Illich I. (1975), Némésis médicale, trad. fse, Paris, Ed. du Seuil.

Keckler S.E, Allen D.T (2006), « Material Reuse Modeling : A Case Study of Water Reuse, in an Industrial Park », Journal of Industrial Ecology, vol 2, n4, p. 79-92.

Kormondy E. J.(1969), Concepts of Ecology. Englewood Cliffs, N.J,Prentice-Hall, Inc. 
Labrune S. (2000), « La valorisation des déchets en énergie à partir des parcs agro-industriels de saules », Mémoire de fin d'études, ISA Beauvais, mars, $128 \mathrm{p}$.

Leontief W. (1936), «Quantitative input and output relations in the economic systems of the United States ", Review of Economics and Statistics, vol 18, n³, p. 105-125.

Lifset R. (2006), “What Next for Industrial Ecology?”, Scientific Workshop “Frontiers of Research in Industrial Ecology', University of Lausanne, November 27 - December 1th, 17 p.

Levine S. (2003), "Comparing products and production in Ecological and Industrial Systems", Journal of Industrial Ecology, vol 7, n², p. 33-42.

Lotka A . (1956),Elements of Mathematical Biology,New York Dover.

Lotka A. (1945), «The Law of Evolution as a Maximal Principle» Human Biology, vol 17, p. 67 - 194.

Marshall A. (1919), Industry and Trade, Mac Millan, New York.

Moll H.C, Noorman K.J et alii (2005), « Pursuing More Sustainable Consumption by Analysing Household Metabolism in European Countries and Cities ", Journal of Industrial Ecology, vol 9, $\mathrm{n}^{\circ}$ 1-2, p. 259-275.

Moolenaar S.W, Lexmond T.M (1998), « Heavy Metal Balances, Part I ”, Journal of Industrial Ecology, vol 2, n 4 , p. 45-60.

Moolenaar S.W, (1999), Moolenaar S.W, Lexmond T.M (1998), « Heavy Metal Balances, Part II ”, Journal of Industrial Ecology, vol 2, n4, p. 45-60.

Nahapétian N. (2002), « L'écologie industrielle, exercice pratique », Alternatives économiques, $\mathrm{n}^{\circ}$ 206, p. 60-63.

Odum E.P. (1971), Fundamentals of Ecology, Philadelphia, W.B. Saunders Company, 3rd ed.

Odum E.P (1983), Basic Ecology, Saunders College Publishing, Philadelphia.

Ozyurt D.B, Realff M.J (2001), “Combining a Geographical Information System and Process

Engineering to Design an Agricultural Industrial System”, Journal of Industrial Ecology, vol 5, n³, p. 13-31.

Peck S. (2001), “When is an Eco-Industrial Park not an Eco-Industrial Park ?", Journal of Industrial Ecology, vol $5, \mathrm{n}^{\circ} 3$, p. 3-5.

Poignant S. (2003), « Rapport d'information sur la politique de soutien au développement des énergies renouvelables ", document d'information de l'Assemblée nationale, n 1153, 79 p.

Powers C.W., Chertow M.R. (1997) «Industrial Ecology », in M.R. Chertow, D.C. Esty (eds) Thinking Ecologically, New Haven, Yale University Press, p. 19-36.

Prigogine I. (1968), Introduction à la thermodynamique des processus irréversibles, Masson, Paris.

Randles S., Berkhout F. (2006), "At the Interface of industrial ecology and innovation studies : some emerging themes", Scientific Workshop 'Frontiers of Research in Industrial Ecology', University of Lausanne, November 27 - December 1th, 13 p.

Rosenqvist H., Aronsson P., Hasselgren K., Perttu K. (1997), "Economics of using municipal wastewater irrigation of willow coppice crops", Biomass and Energy, vol 12, n¹, p. 1-8.

Schulze P. (1996), Engineering with Ecological Constraints, National Academy Press.

Smouts M.C, Antoine S., Beauchamp A., Bourg D. (2005), Le développement durable, les termes du débat, Dalloz. 
Svalov Municipality (1998), Kageröd Recycling Projet, Department of civil works, Sweden, 3 p.

Tilley D.R (2003), “Industrial Ecology and Ecological Engineering : Opportunities for Symbiosis”, Journal of Industrial Ecology, vol 7, n 2, p. 13-32.

Tukker A., Cohen M.J, Zoysa U. et alii (2006), « The Oslo Declaration on Sustainable Consumption", Journal of Industrial Ecology, vol 10, n², p. 9-14.

Vivien F. D. (1994), "Economie et écologie", La découverte

Vivien F.-D. (2000), « Industrielle ou politique ? Quelle écologie pour le développement durable? » in Bourg D., Erkman S. (eds) Industrial Ecology and Sustainability : Proceedings, Troyes/ ICAST.

Vivien F.D (2002), « Rencontre du troisième type... d'écosystème ou quand l'écologie devient industrielle », Colloque de Dunkerque, 8 p.

Vivien F.D (2005), Le développement soutenable, La Découverte.

Vivien F.D (2006), “L'écologie industrielle : une critique du point de vue de l'économie politique”, Scientific Workshop 'Frontiers of Research in Industrial Ecology', University of Lausanne, November 27 - December 1th, $22 \mathrm{p}$.

Watt K.E.F (1973), Principles of Environmental Sciences, Mc Graw Hill, New-York.

Wells P., Orsato R.J (2005), "Redesigning the Industrial Ecology of the Automobile", Journal of Industrial Ecology, vol 9, n³, p. 15-30.

Zhu Q., Lowe E.A, Wei Y.A, Barnes D. (2007), “Industrial Symbiosis in China”, Journal of Industrial Ecology, vol 11, n¹, p. 31-42.

\section{NOTES}

1. F.D Vivien (2005) parle de « développement soutenable».

2. Pour une comparaison écologie industrielle - écologie politique, voir Dannequin et al. (2000), Vivien (2006).

3. Par ses relations avec le monde industriel et plus particulièrement l'industrie automobile (Tukker et Cohen (2004) ont analysé le concept d'écologie industrielle chez Ford), l'écologie industrielle est considérée par F.D Vivien (2006, p. 9) comme pouvant être porteuse "d'un néotaylorisme ou d'un néo-fordisme ».

4. L'écologie industrielle ne se limite pas au secteur automobile. Elle touche toute la communauté des ingénieurs. On peut ainsi citer l'ouvrage de Graedel et Allenby (1995), Industrial Ecology. L'un est «membre distingué » du staff technique, l'autre est vice-président de la recherche « Technology andEnvironment » de la société AT \& T Bell Laboratories.

5. Tilley (2003) distingue deux disciplines qu'il nomme "Industrial ecology» et "Ecological Engineering ".

6. Les deux principes de la thermodynamique (échange et transformation de l'énergie et de la matière) ont joué un rôle fondamental dans la construction de l'écologie.

7. Ce qui caractérise la typologie des écosystèmes d'Eugène Odum, c'est l'alternative entre l'énergie solaire et l'énergie d'origine humaine. On distingue les écosystèmes naturels «non subventionnés » alimentés par l'énergie solaire ; les écosystèmes alimentés par l'énergie solaire et «subventionnés » par d'autres écosystèmes; les écosystèmes alimentés par l'énergie solaire mais subventionnés par l'homme (exemple des agro-écosystèmes); les écosystèmes urbanoindustriels alimentés par les combustibles. 
8. Les écosystèmes sont soumis à 40 principes. Le principe 0 correspond à une description, une analyse et une gestion possible des écosystèmes en fonction des paramètres ressources (dans une vision classique, le fonctionnement est assuré par la matière, l'énergie, l'espace, le temps, la diversité). Les principes I et II sont rattachés aux deux lois de la thermodynamique...

9. Le rapport « Halte à la croissance » (1973)s'appuiera sur l'approche systémique.

10. Huppes et Ishikawa $(2005$, p. 27) ont identifié quatre types d'éco-efficience : «The first two are environnemental productivity and its inverse, environnemental intensity of production... The second pair, environnemental improvement cost and its inverse, environnemental costeffectiveness are defined from an environnemental improvement measures point of view".

11. Esty et Porter considèrent que "the systems perspective that industrial ecology promotes can help companies find ways to add value or reduce costs both within their own production processes and up and down the supply chain" ([1998], p. 35). L'écologie industrielle propose ainsi une approche collective et systémique qui va à l'encontre des schémas traditionnels (agents économiques individualistes, logique concurrentielle).

12. Andrew (2000) cherche à construire les fondements micro de l'écologie industrielle.

13. DiMaggio et Powell définissent le champ organisationnel « as a recognized aera of institutional life : key suppliers, resource and product consumers, regulatory agencies, and other organisations that produce similar services and products" (1983, p. 148).

14. Dans un écosystème parfait, le seul input est l'énergie solaire. Toutes les autres matières sont recyclées biologiquement dans le sens où les déchets de chaque espèce sont la nourriture d'une autre espèce.

15. Frosh et Galloupolos (1989, p. 107) soulignaient déjà que « la prise de conscience écologique devrait amener les pays en voie de développement à effectuer des sauts technologiques, passage direct à des méthodes plus modernes ".

16. Daniels $(2001,2002)$ a comparé les dimensions physiques de neuf systèmes économiques afin de quantifier les flux et les stocks de matière associés à l'activité humaine.

17. Fischer-Kowalski, Hüttler (1998, p. 107) ont proposé une classification du métabolisme en retenant trois niveaux: le modèle de référence (écosystème biologique, écosystème socioéconomique), le niveau du système (global, national, régional, fonctionnel, temporel) et les types de flux (matières, énergies, substances).

18. Moll, Norman, Kok et alii (2005) ont analysé le concept de "Household metabolism» en l'introduisant dans des modèles de consommation. L'analyse des différents types de ménages (citadins européens), à partir des besoins (directs et indirects) d'énergie, permettrait de définir un modèle de consommation plus 'soutenable'.

19. En France, c'est la Loi sur les Nouvelles Régulations Economiques (LNRE) de 2001 qui impose aux entreprises de publier annuellement un bilan environnemental.

20. Selon Nicholas Georgescù-Roegen (1978, p 353), la thermodynamique devient un des éléments de la constitution de la valeur économique : « la thermodynamique ... est au fond une physique de la valeur économique, c'est à dire une science qui étudie les qualités physiques qui confêrent en général à certaines choses une valeur pour l'homme ".

21. « La loi de l'entropie est la racine de la rareté économique. Si cette loi n'existait pas, nous pourrions réutiliser l'énergie d'un morceau de charbon à volonté, en le transformant en chaleur, cette chaleur en travail, et ce travail à nouveau en chaleur » (Georgescù-Rogen 1995, p 51).

22. Christensen $(2006$, p. 2) parle de «Collaboration between different industries for mutual economic and environmental Benefit ».

23. Christensen (2005) a relativisé ces critiques en rappelant que l'engagement dans la durée donnait une certaine visibilité aux partenaires et que les actions étaient basées sur des contrats commerciaux ordinaires (en cas de rupture du contrat, des clauses permettent aux partenaires d'avoir du temps pour trouver un autre fournisseur).

24. « Communication is more important than technology» (Christensen 2006, p. 48). 
25. Chertow (2007, p. 13) a identifié ce que l'on pourrait appeler des pré-conditions à la symbiose (collaboration, effets de synergie offerts par la proximité géographique), il s'agit notamment derépertorier les externalités positives (partage d'une ressource ou d'une matière, solution technique...).

26. On assiste depuis 1993 à une floraison de parcs éco-industriels, principalement aux Etats-Unis (Devens, Massachusetts ; Baltimore, Maryland ; Cape Charles, Virginie...) ; Canada (Halifax) ; PaysBas (Rotterdam); France (Parc industriel de la plaine de l'Ain, Ecopal de Dunkerque); Suède (Landskrona) ; Philippines (Prime Projet) ; Chine (TEDA)... L'écologie industrielle est ainsi perçue comme une stratégie permettant d'accroître la compétitivité économique (Esty, Porter, 1998).

27. Kageröd est un village appartenant à la municipalité de Svalöf (Suède). Il réalise le traitement d'une partie des eaux domestiques de ses 1500 habitants ainsi que les effluents d'une entreprise fabricant de la poudre de lait (correspondant aux eaux usées de 6000 habitants).

28. Le passage des machines en conditions humides peut entraîner un tassement du sol.

29. Pour approvisionner une chaufferie, il faut un silo de stockage, une vis sans fin et une chaudière avec toutes les options indispensables (décendrage, filtre à fumées...). Cette chaudière fournit ainsi de la chaleur en fonction des besoins du consommateur.

30. Le centre de tri des colis des 3 Suisses est chauffé à partir du bois produit par cinq hectares de TTCR. Cette société dispose d'une telle surface sur sa réserve foncière. Elle a planté près de 100000 saules qui fournissent 250 tonnes de bois de chauffage tous les trois ans. L'ADCE se charge du suivi technique des parcelles (Rapport de l'ADCE [1998]).

31. Par contre,les coûts d'investissement et d'aménagements de la chaufferie ou d'un gazogène sont importants.

32. Voir le « Rapport d'information déposé en application de l'article 145 du Règlement par la Commission des affaires économiques, de l'environnement et du territoire sur la politique de soutien au développement des énergies renouvelables » de Serge Poignant, Assemblée nationale (2003), $\mathrm{n}^{\circ} 1153,79$ p.

33. Située à Villeneuve d'Asq, la station d'épuration de la Communauté urbaine de Lille et de sa région traite les eaux usées aux niveaux primaire et secondaire. Elle expérimente, depuis 1998, un traitement tertiaire de ses eaux usées grâce à la micro-irrigation des saules.

34. Dans le cadre du programme FAIR (Aide européenne pour les projets innovants dans le domaine de l'environnement), la société LEROUX SA s'est associée à l'ADCE pour réaliser l'épandage de ses effluents sur les TTCR de saules. La biomasse produite est récoltée tous les deux ou trois ans pour servir de combustibles dans une chaudière à bois appartenant à une association d'aide aux handicapés (Rapport de l'Association pour le Développement des Cultures énergétiques, ADCE 1998).

\section{RÉSUMÉS}

L'écologie industrielle, définie par Robert Frosch (1995) comme «l'ensemble des pratiques destinées à réduire la pollution industrielle ", nous amène à penser que l'écosystème industriel peut être un véritable vecteur du développement durable. L'ingénierie écologique et l'écotechnologie recommandent aux industriels de procéder à un ensemble d'opérations de rationalisation de la production (optimisation des consommations énergétiques et matérielles, minimisation des déchets à la source,réutilisation des rejets pour servir de matières premières à d'autres processus de production). Les symbioses industrielles et les parcs éco-industriels sont généralement 
présentés comme des modèles de rationalisation industrielle et des illustrations tangibles du développement durable.

Industrial ecology is defined by Robert Frosch (1995) as practices intended to reduce industrial pollution. That leads us to think industrial ecosystem as a vector of sustainable development.Ecological engineering and ecotechnology recommend managers to rationalize the production process (optimization of material consumptions, minimization of bads...). Industrial symbiosis and industrial parks are generally presented as models of industrial rationalization and tangible illustrations of the sustainable development.

\section{INDEX}

Mots-clés : écologie industrielle, métabolisme, symbiose, parc-éco industriel, écotechnolologie, taillis à très courte rotation

Keywords : industrial ecology, ttcr, metabolism, symbiosis, ecotechnology, industrial park

\section{AUTEURS}

\section{ARNAUD DIEMER}

Arnaud Diemer est Maître de Conférences à l'IUFM d'Auvergne. Chercheur rattaché au GRESE « Groupe de recherche en épistémologie et socio-économie » de l'université Paris I et à OMI « organisations, marchés, institutions » de l'université de Reims. Thèmes de recherche : articulation dans l'histoire des sciences entre la science économique et les sciences physiques; histoire de la pensée économique ; perspectives économiques du développement durable. Travaux sur l'écologie industrielle et le développement durable réalisés lors de colloques ou pour le compte de revues (Cahiers du Ceras, Université de Reims). Contact : 15 rue Gambetta, 58000 Nevers, diemera@aol.com

\section{SYLVÈRE LABRUNE}

Sylvère Labrune est Ingénieur agricole de l'Institut Supérieur Agricole de Beauvais. Thèmes de recherche : articulation dans l'histoire des sciences entre la science économique et les sciences physiques ; histoire de la pensée économique ; perspectives économiques du développement durable. Travaux sur l'écologie industrielle et le développement durable réalisés lors de colloques ou pour le compte de revues (Cahiers du Ceras, Université de Reims). 